First publ. in: Journal of Low Temperature Physics 138 (2005), 1-2, pp. 355-360

\title{
Equilibrium helium films under the influence of surface roughness
}

\author{
J. Klier ${ }^{1}$, M. Zech ${ }^{1}$, A. Fubel ${ }^{1}$, P. Leiderer ${ }^{1}$, and V. Shikin ${ }^{2}$ \\ 1 Department of Physics, University of Konstanz, D-78457 Konstanz, Germany \\ 2 ISSP, 142432 Chernogolovka, Moscow District, Russia
}

Equilibrium helium films adsorbed on solid substrates are investigated. Due to their thickness these films are mainly in the retardation regime where the influence of the roughness of the substrates, $\delta(x)$, can be strong enough to be observed. For the definition of $\delta(x)$ we use a simple corrugation model. This model is supported by experimental results using the surface plasmon resonance technique to determine the thickness of helium films grown on different Ag surfaces.

PACS number: 67.70.+n, 68.15.+e, 68.43.- $h, 68.55 .-a$

\section{INTRODUCTION}

The thickness of a liquid film grown under complete wetting conditions on a substrate is an important parameter in many areas of condensed matter physics, especially for surface science studies. Under thermodynamical equilibrium conditions the thickness of a liquid film can be changed by variation of its vapour pressure, $P$. When there is no saturation of the vapour pressure the thickness of the adsorbed film is usually very thin (a few monolayers). In the other case, i.e., when there is coexistence with its saturated vapour pressure, $P_{\text {sat }}$, the film thickness, $d$, is dependent on the distance $h$ of the bulk liquid level to the surface of the substrate, see Fig. 1a.

The existing description of the dependence $d(h) \equiv d_{\mathrm{vdW}}(h)$, under ideal saturation conditions (i.e., substrate is flat and infinite) [1-4], deals with the competition between van-der-Waals (vdW) and gravitational forces,

$$
\frac{\Delta C_{3} k_{\mathrm{B}}}{d_{\mathrm{vdW}}^{3}}\left(1+\frac{d_{\mathrm{vdW}}}{d^{\star}}\right)^{-1}=-m g h \quad \text { for } \quad \kappa|h|>1 .
$$

Here, $\Delta C_{3}$ is the Hamaker constant, $d^{\star}$ is the liquid film thickness at which 
J. Klier, M. Zech, A. Fubel, P. Leiderer, and V. Shikin
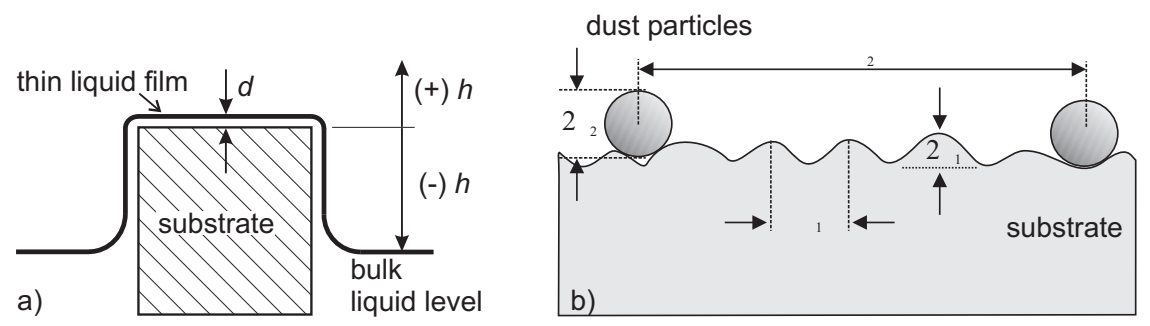

Fig. 1. a) A thin liquid film with thickness $d$ is completely wetting a horizontal substrate and is in coexistence with $P_{\text {sat }}$ (i.e., in presence of bulk liquid). The distance between substrate and bulk liquid level is $(-) h$. b) Schematic sketch of a surface showing intrinsic and extrinsic roughness having, e.g., the periodicity $\lambda_{1}$ and amplitude $\delta_{1}$, and $\lambda_{2}$ and $\delta_{2}$ respectively. The extrinsic roughness is mainly due to surface contamination by dust particles.

the transition from the non-retarded to the retarded regime occurs, $m$ is the mass of an adsorbent atom (or molecule), $g$ is the acceleration due to gravity, and $\kappa=\sqrt{\rho g / \sigma_{\mathrm{lv}}}$ is the inverse capillary length, where $\rho$ is the density and $\sigma_{\mathrm{lv}}$ the surface tension of the adsorbed liquid. The inequality $\kappa|h|>1$ avoids the divergency of $d$ when $h \rightarrow 0$. The definition of $h$ corresponds to Fig. 1a. The interpolation between non-retarded and retarded regime was proposed by Putterman [3].

Such an ideal dependence in the film growth is confirmed by experiments [5] where liquid ${ }^{4} \mathrm{He}$ has been used as adsorbent and cleaved crystals as substrates, which should provide an atomically flat surface. There are many additional measurements (see Ref. [4]), where different preparation techniques and materials have been used. However, we do not know any example in literature, where the contribution of the substrate roughness to the thickness of saturated van-der-Waals films is discussed quantitatively. So far, the existing discussions deal with liquid films under unsaturated vapour pressure conditions only (see [6-9]).

Here we investigate the influence of substrate roughness on the thickness of an adsorbed liquid film, which is in coexistence with its saturated vapour pressure. We derive a model which describes this influence and compare it with measurements of the thickness of liquid ${ }^{4} \mathrm{He}$ films adsorbed on $\mathrm{Ag}$ substrates with different roughness.

\section{THEORETICAL DESCRIPTION}

We consider surface perturbations which can, on one hand, arise due to intrinsic substrate roughness (e.g. on gold and silver surfaces) and, on the 


\section{Equilibrium helium films - influence of surface roughness}

other hand, by accumulation of dust particles (see Fig. 1b). This statement is supported by AFM measurements which were carried out on Au surfaces used in cryogenic experiments [10]. Since we assume these substrates not to be necessarily simple periodic, we treat each perturbation, $\delta_{i}(x)$, as a fourier sum of $j$ components. Therefore, we get for the shape of the substrate, $\delta(x)=\sum_{i} \delta_{i}(x)$, the following relation:

$$
\delta(x)=\sum_{i=1}^{N} \sum_{j=1}^{\infty} \delta_{i j} \cos \left(\frac{j \pi}{\lambda_{i}} x\right), \quad \int \delta_{i}(x) \mathrm{d} x=0 .
$$

Furthermore, we assume for simplicity $\delta_{i}(x)=\delta_{i}(-x)$. The same relation is applied for the $y$-direction without a correlation between $x$ and $y$. The factor $N$ reflects the number of possible surface perturbations. In the discussion below, it will be either $N=1$ or $N=2$ (depending on the substrate).

The profile $d(x, h)$ of a liquid film on a rough substrate can now be calculated from generalisation of Eq. (1), see Refs. [11,12],

$$
\sigma_{\mathrm{lv}} V_{\mathrm{a}} \frac{\nabla^{2} d}{\left[1+(\nabla d)^{2}\right]^{3 / 2}}+\frac{\Delta C_{3} k_{\mathrm{B}}}{(d-\delta)^{3}}\left(1+\frac{d-\delta}{d^{\star}}\right)^{-1}=-m g h .
$$

The thickness $d(x, h)$ is defined to have the same zero level as the roughness $\delta(x) . V_{\mathrm{a}}$ is the volume of a single adsorbent atom (or molecule). The local approximation for the non-uniform problem, i.e., the second term on the l.h.s. of Eq. (3) is not correct in general. Therefore, we have to take into account a non-local correction via renormalisation of $\delta_{i j}$ (see $[12,13]$ ),

$$
\delta_{i j} \rightarrow \delta_{i j} \frac{q d}{2} K_{2}(q d), \quad q=\frac{2 \pi}{\lambda_{i}},
$$

where $K_{2}(x)$ is the modified Bessel-function

$$
K_{2}(x)=\left\{\begin{array}{cl}
2 / x & , x \rightarrow 0 \\
(\pi / 2 x)^{1 / 2} \mathrm{e}^{-x} & , x \gg 1
\end{array} .\right.
$$

If $d^{\star}<d$ and $\nabla d<1$ (which corresponds to our experimental situation, where $\delta_{i} \ll \lambda_{i}$ ), Eq. (3) can be simplified to

$$
\sigma_{\mathrm{lv}} V_{\mathrm{a}} \frac{\mathrm{d}^{2} d}{\mathrm{~d} x^{2}}+\frac{\Delta C_{3} k_{\mathrm{B}} d^{\star}}{(d-\delta)^{4}}=-m g h .
$$

After introduction of $d(x, h)=d_{\mathrm{vdW}}(h)+\zeta(x, h)$ and simple manipulation with (5), we have

$$
\delta(x)-\zeta(x, h)=d_{\mathrm{vdW}}(h)\left[1-\left(\frac{1}{1-R \frac{\mathrm{d}^{2} \zeta}{\mathrm{d} x^{2}}}\right)^{\frac{1}{4}}\right],
$$




\section{J. Klier, M. Zech, A. Fubel, P. Leiderer, and V. Shikin}

where $d_{\mathrm{vdW}}(h)^{4}=-\Delta C_{3} k_{\mathrm{B}} d^{\star} / m g h$ and $\zeta(x)$ is the additional film thickness due to surface roughness. $R=-\sigma_{\mathrm{lv}} / \rho g h$ is the Laplace radius.

It can be shown, that the term $R \frac{\mathrm{d}^{2} \zeta}{\mathrm{d} x^{2}}$ from Eq. (6) is less than 1 in all interesting intervals of $h$. We can use this property for the simplification of this equation. Assuming

$$
\zeta(x, h)=\sum_{i=1}^{N} \frac{\zeta_{i 0}}{2}+\sum_{j=1}^{\infty} \zeta_{i j} \cos \left(\frac{j \pi}{\lambda_{i}} x\right),
$$

we get for the expansion of Eq. (6) to the lowest non-linear terms $(j=1$; which turns out to be sufficient to describe our experimental data, see below)

$$
\begin{gathered}
\zeta_{i 1}=\frac{\delta_{i 1}}{1+\frac{1}{4} \frac{\pi^{2}}{\lambda_{i}^{2}} R d_{\mathrm{vdW}}} \\
\zeta_{i 0} \equiv<\zeta_{i}>=\frac{5}{32} R^{2} d_{\mathrm{vdW}}\left[\zeta_{i 1}^{2} \frac{\pi^{4}}{\lambda_{i}^{4}}+\ldots\right] .
\end{gathered}
$$

To be consistent with the non-local predictions, the results (8) and (9) have to be connected, see Eq. (4), using $\delta_{i j} \rightarrow \delta_{i j} \frac{q d}{2} K_{2}(q d)$. The obtained result (9) is the necessary correction describing the change in $d(h)$ due to a surface corrugation with finite amplitude $\delta(x) \neq 0$.

\section{EXPERIMENTAL OBSERVATIONS}

To investigate the influence of substrate roughness on the equilibrium thickness of an adsorbed film, and to verify the above predictions, we have measured the thickness of a growing liquid ${ }^{4} \mathrm{He}$ film on different silver substrates. Hereby the thickness of the adsorbed helium layer was varied over a wide range by varying the distance $-h$ between the liquid helium level and the substrate, which is measured with a cylindrical capacitor. The experiments are performed at $1.4 \mathrm{~K}$. As experimental technique we used a surface plasmon resonance setup which allows the film thickness measurement of the adsorbed helium layer with a resolution of about $1 \AA$, see Ref. [14]. For details of the experimental setup see, e.g., Ref. [15].

In Fig. 2 we show the growth of a helium film for two different Agsubstrates starting from a bulk level $(h \approx-6.2 \mathrm{~cm})$ and finishing at the level $h \leq-0.1 \mathrm{~cm}$, corresponding to the requirement $d \ll-h$. One of these substrates is assumed to be very smooth, since it was prepared under relatively clean conditions. This assumption is confirmed by the good agreement between the film thickness data and Eq. (1), which describes the adsorption on ideal surfaces. Only a small extrinsic roughness contribution is necessary 


\section{Equilibrium helium films - influence of surface roughness}
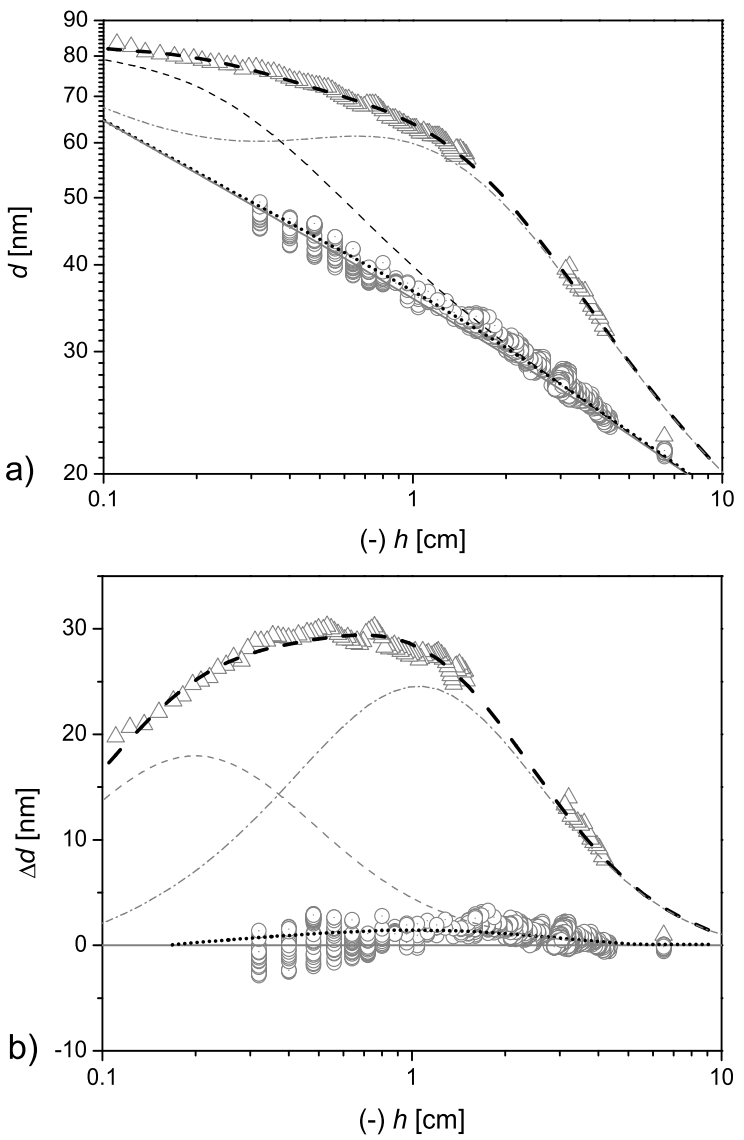

Fig. 2. a) Film thickness dependence of adsorbed helium on two different $\mathrm{Ag}$ substrates $(\odot$ and $\triangle$ ). The solid line describes the adsorption on an ideal flat surface using $\Delta C_{3} \approx 3000 \times 10^{-30} \mathrm{~K} \mathrm{~m}^{3}$. The dashed line is computed for a rough substrate, the dotted line is for a smooth substrate, see text. b) Difference between measured and calculated ${ }^{4} \mathrm{He}$ film thickness for strong roughness (dashed line) and weak roughness (dotted line). The thin dashdotted and dashed line in a) and b) show the contribution of each individual surface roughness denoted by $\delta_{11}, \lambda_{1}$ and $\delta_{21}, \lambda_{2}$, respectively.

to explain the experimental data within the error of measurement, corresponding to $N=1$, where $N$ is from Eq. (2). The second substrate has been exposed to air for a longer period, causing contamination by dust particles, and is therefore assumed to have a pronounced surface roughness. Indeed, the film thickness data show a large deviation from Eq. (1). Therefore, the theoretical description for this substrate has to include the influence of roughness as presented in Eq. (9) and Fig. 1b. The dashed line in Fig. 2 (for the 


\section{J. Klier, M. Zech, A. Fubel, P. Leiderer, and V. Shikin}

rough substrate) has been computed using the parameters $\delta_{11}=77_{-2}^{+3} \mathrm{~nm}$, $\lambda_{1}=2.6_{-0.2}^{+0.1} \mu \mathrm{m}, \delta_{21}=83_{-3}^{+4} \mathrm{~nm}$, and $\lambda_{2}=7.6_{-0.3}^{+0.4} \mu \mathrm{m}$ (best fit). The dotted line (for the smoother substrate) has been calculated using $\delta_{11}=15_{-3}^{+5} \mathrm{~nm}$ and $\lambda_{1}=2.7_{-0.5}^{+0.3} \mu \mathrm{m}$. In both cases, good agreement with the experimental data is found. The values obtained from the AFM studies mentioned in the beginning [10] are similar to those used for $\delta_{i j}$ and $\lambda_{i}$ within our analysis.

\section{CONCLUSIONS}

We have investigated the growth dependence of adsorbed liquid helium on Ag-substrates with different roughness as function of the distance between the bulk liquid level and the surface of the substrate, which is above the bulk level. Calculations of the thickness of such films taking the surface roughness of the substrate into account are presented. These calculations contain both the influence of an intrinsic roughness of the underlaying substrate and an extrinsic roughness as produced, e.g., by the contamination of the substrate with dust particles. The calculations are confirmed by measurements of equilibrium saturated liquid helium films adsorbed on silver.

We thank V. Iov and F. Schletterer for help in the experiments. This work was supported by the DFG-Schwerpunkt 'Wetting and Structure Formation at Interfaces' under Kl 1186/1 and grant RFBI 030216121.

\section{REFERENCES}

1. H.B.G. Casimir and D. Polder, Phys. Rev. 73, 360 (1948).

2. I.E. Dzyaloshinskii, E.M. Lifshitz, and L.P. Pitaevskii, Adv. Phys. 10, 165 (1961).

3. S. Putterman, Superfluid hydrodynamics, North Holland series in low temperature physics, Amsterdam (1974).

4. see e.g., L.W. Bruch, M.W. Cole, and E. Zaremba, Physical Adsorption: Forces and Phenomena, Clarendon Press, Oxford (1997); and references therein.

5. E.S. Sabisky and C.H. Anderson, Phys. Rev. A 7, 790 (1973).

6. P. Pfeifer and D. Avenir, J. Chem. Phys. 79, 3558 (1983).

7. P. Pfeifer, Y. Wu, M. Cole, and Y. Krim, Phys. Rev. Lett. 62, 1997 (1989).

8. V. Panella and J. Krim, Phys. Rev. E 49, 4179 (1994).

9. K. Mecke and J. Krim, Phys. Rev. B 53, 2073 (1996).

10. A. Faustein, Diploma thesis, pp. 41-42 (2004).

11. L.D. Landau and E.M. Lifshitz, Hydrodynamics, Akademie Verlag Berlin (1991).

12. M. Robbins, D. Andelman, and J. Joanny, Phys. Rev. A 43, 4344 (1991).

13. Yu. P. Monarkha, Sov. J. Low Temp. Phys. 18, 145 (1992).

14. D. Reinelt, J. Klier, and P. Leiderer, J. Low Temp. Phys. 113, 805 (1998).

15. V. Iov, J. Klier, and P. Leiderer, J. Low Temp. Phys. 126, 367 (2002). 